\title{
PREDICTING THE THERMAL CONDUCTIVITY OF EPOXY/MWCNT COMPOSITES USING ANALYTICAL MODELLING
}

\author{
Mohammed Ghuzi \\ Department of Chemical and Materials Engineering. \\ University of Alberta \\ Edmonton, AB, Canada \\ ghuzi@ualberta.ca
}

\author{
Pierre Mertiny \\ Department of Mechanical Engineering. \\ University of Alberta \\ Edmonton, AB, Canada \\ pmertiny@ualberta.ca
}

\begin{abstract}
Polymer nanocomposites have opened a new path for multifunctional materials. In particular, carbon nanotubes have the potential to be used in various applications. This study focused on the evaluation of thermal conductivity of epoxy/carbon nanotube composites using analytical modeling. The influence of the filler content, the geometry, the size, and the aspect ratio on thermal conductivity of the composite were discussed within the context of the studied models.
\end{abstract}

Keywords - polymer nanocomposites; multi-walled carbon nanotubes; thermal condcutivity; analytical modelling

\section{INTRODUCTION}

Polymer-based composites (PC) have received significant attention due to their promising potential in various applications. They are rapidly replacing traditional inorganic materials such as metals, and natural materials such as wood due to their superior physical and mechanical properties [1]. One of the promising applications involves the use of PC materials (e.g. thermoset or thermoplastic matrices with glass or carbon fiber reinforcement) in different structural applications such as aircrafts, ships and automobiles [2]. However, PC are usually flammable and have comparatively low resistance to fire. As such, a critical safety issue may arise when PC get exposed to fire resulting in failure and collapse of a structure, and hence causing injuries or even fatalities. Therefore, a research thrust has been immerging to improve the flammability properties of PC using nanofillers such as carbon nanotubes (CNT). Nanofillers with suitable morphology, distribution and dispersion act in the condensed phase akin to char forming flame retardants, that is, they create a thermal insulation and mass transport barrier for the underlying polymer, hence mitigating the creation and escape of gaseous fuel for combustion. In addition, nanofillers may greatly increase the viscosity of the polymer decomposition phase, thus inhibiting flow and drip-off, reducing the decomposition area available for combustion. Kashiwagi et al. [3] used multiwalled carbon nanotubes (MWCNT) as a fire retardant additive in polypropylene. They found that MWCNT significantly enhance the thermal stability of polypropylene in nitrogen atmosphere at high temperatures by greatly reducing the heat release rate of the polypropylene. However, in a different study, it was reported that an increase in MWCNT concentration resulted in an increase in a peak heat release rate due to an increase in thermal conductivity of the nanocomposites [4]. While polymers have low thermal conductivity ranging approximately from $0.2 \mathrm{~W} \cdot \mathrm{m}^{-1} \mathrm{~K}^{-1}$ for amorphous polymers to $0.5 \mathrm{~W} \cdot \mathrm{m}^{-1} \mathrm{~K}^{-1}$ for highly crystalline polymers, CNT possess a thermal conductivity that is four order of magnitude higher $\left(2000-6000 \mathrm{~W}^{-1} \cdot \mathrm{K}^{-1}\right)$ [5]. Reducing the thermal insulation properties inherent to polymers may thus lead to reduced ignition times [4]. It is therefore necessary to gain a thorough understanding of the thermal response of nanocomposites, such as heat release rate, time of ignition and heat conduction, to evaluate their flammability and thus safety [2]. One of the main thermalphysical properties that controls the thermal response is thermal conductivity.

Many studies reported that the incorporation of CNT into a polymer matrix can lead to a certain enhancement of thermal conductivity of the CNT/polymer nanocomposites. Thermal conductivity is a phonon based mechanism influenced by many factors such as the thermal conductivity of each constituent, the shape, volume fraction, and the dispersion of nanofillers into the polymer matrix, as well as the interfacial thermal resistance between the filler and the polymer [6]. Manipulating one or more of these factors to observe the effect on the composite thermal conductivity might require a multitude of experiments. It is therefore imperative to find methods for estimating the thermal conductivity of the CNT/polymer nanocomposites and hence limit the number of experiments. Among these methods, analytical models supply closed-form expressions for the composite thermal conductivity. However, numerous analytical models have been proposed since the $19^{\text {th }}$ century, and selecting the right model is not always straightforward.

The present paper reports on the modelling the thermal conductivity of epoxy composites filled with low content of MWCNT. Analytical models available in the technical literature were employed for this purpose, and their associated results were contrasted to experimental data from published studies. The aim of this work is explore the effectiveness of selected thermal conductivity prediction models and provide a perspective on the aforementioned factors and their significance when estimating the thermal conductivity of epoxy/MWCNT composites. 


\section{REVIEW OF LITERARTURE MODELS}

A variety of analytical models have been developed to predict the effective thermal conductivity of a composite material. They range from simple expressions (e.g. rule of mixture models) to rather complex expressions, depending on the factors taken into consideration. In this section, the most expedient models, based on the authors' point of view, are discussed. The following notations are used: $K_{\mathrm{c}}, K_{\mathrm{m}}, K_{\mathrm{f}}$ are correspondingly the thermal conductivities (through-thickness direction) of the composite, polymer matrix and filler; and $V_{\mathrm{f}}$ is the filler volume fraction.

\section{A. Maxwell model [7]:}

The Maxwell expression is one of the early models proposed to predict the thermal conductivity of a composite material. The model was developed in 1904 and it describes a system of dilute spherical particles embedded in a continuous matrix. The model neglects the thermal interactions between the filler particles and only accounts for the thermal conductivity of the constituents and the volume fraction of the dispersed spherical fillers as follows:

$$
K_{\mathrm{c}}=K_{\mathrm{m}}\left(1-\frac{3 V_{\mathrm{f}}}{\frac{K_{\mathrm{f}}-2 K_{\mathrm{m}}}{K_{\mathrm{f}}-K_{\mathrm{m}}}-V_{\mathrm{f}}}\right)
$$

\section{B. Hamilton and Crosser model [8]:}

Hamilton and Crosser improved the Maxwell expression by taking into account the effect of the geometry of the filler. They showed that for non-spherical fillers, there is a drastic increase in thermal conductivity of the composite provided that the discrepancy in thermal conductivity of the continuous phase and the dispersed phase is an order of 100 or more. Thus, they introduced an empirical shape factor, $n$, that accounts for such effect as follows:

$$
K_{\mathrm{c}}=K_{\mathrm{m}}\left[\frac{K_{\mathrm{f}}+(n-1) K_{\mathrm{m}}-(n-1) V_{\mathrm{f}}\left(K_{\mathrm{m}}-K_{\mathrm{f}}\right)}{K_{\mathrm{f}}+(n-1) K_{\mathrm{m}}+V_{\mathrm{f}}\left(K_{\mathrm{m}}-K_{\mathrm{f}}\right)}\right]
$$

The shape factor, $n$, can be calculated using the following:

$$
n=\frac{3}{\gamma}
$$

where $\gamma$ is the sphericity and it is defined as the ratio of the surface area of a sphere (with the same volume as the given particle) to the surface area of the particle.

\section{Lewis-Nielsen model [9]:}

Even though the Lewis-Nielsen model does not consider the Kapitza or interface thermal resistance (ITR) between a filler particle and its surrounding polymer matrix, it was found to yield results that are in good agreement with measurements [9]. The model accounts for a wide range of filler and nanocomposite morphologies and can be described as follows:

$$
K_{\mathrm{c}}=\frac{1+\left(\frac{K_{\mathrm{f}} / K_{\mathrm{m}}-1}{K_{\mathrm{f}} / K_{\mathrm{m}}+A}\right) A V_{\mathrm{f}}}{1-\left(\frac{K_{\mathrm{f}} / K_{\mathrm{m}}-1}{K_{\mathrm{f}} / K_{\mathrm{m}}+A}\right) \beta V_{\mathrm{f}}}
$$

where $A$ is the shape coefficient of the filler particles and it depends on the particle's aspect ratio; $\beta$ accounts for the type of packing of the particles and can be calculated by Eq.(5).

$$
\beta=1+\left(\frac{1-V_{\mathrm{m}}}{V_{\mathrm{m}}^{2}}\right) V_{\mathrm{f}}
$$

where $V_{m}$ is the maximum packing fraction of the filler.

\section{Model by Deng et al. [10]:}

Deng et al. developed a specific analytical model for predicting the thermal conductivity of CNT composites with low CNT loading. The model takes into consideration the effects of the thermal conductivity anisotropy, aspect ratio, non-straightness, interfacial thermal resistance, interaction, and either a random or aligned CNT distribution. The model can be written in the following form:

$$
K_{\mathrm{c}}=K_{\mathrm{m}}\left[\left(\frac{1}{3} \frac{\theta}{\frac{K_{\mathrm{m}}}{\theta K_{33}^{\mathrm{cs}}}+H(\theta p)}\right) V_{\mathrm{f}}+1\right]
$$

where

$$
\begin{gathered}
K_{33}^{\mathrm{cs}}=\frac{K_{33}^{\mathrm{c}}}{\left(1+\frac{2 R_{\mathrm{k}} K_{33}^{\mathrm{c}}}{L}\right)} \\
H(\theta p)=\frac{1}{(\theta p)^{2}-1}\left[\frac{\theta p}{\sqrt{(\theta p)^{2}-1}} \ln (\theta p\right. \\
\left.+\sqrt{\left.(\theta p)^{2}-1\right)}-1\right]
\end{gathered}
$$

where is $\theta$ is the straightness ratio and is given by $\theta=L^{\text {ce }} / L$; $L^{\text {ce }}$ is the equivalent average straight length of carbon nanotubes; $L$ is the average twisted length of carbon nanotubes; $p$ is the aspect ratio and is given by $p=L / d$ where $d$ is the average carbon nanotube diameter; $K_{33}^{\text {cs }}$ is the equivalent longitudinal thermal conductivity; $K_{33}^{\mathrm{c}}$ is the axial thermal conductivity of the CNTs; $R_{\mathrm{k}}$ is the ITR.

\section{VALIDATION AND VERFICATION OF MODELS}

For the present study, experimentally determined thermal conductivity data for epoxy/MWCNT nanocomposites as a function of the filler volume fraction were obtained from the technical literature. The literature data collected for epoxy/MWCNT nanocomposites varied in their properties due to differences in the constituent materials used in composite fabrication and possibly also due to the measurement methods, the thermal conductivities of the composite were normalized with their respective thermal conductivity of neat epoxy. Table 1 lists the different epoxy/MWCNT nanocomposite systems that were adopted for this study. 
TABLE 1. SUMMARY OF EPOXY/MWCNT NANOCOMPOSITES TAKEN FROM TECHNICAL LITERATURE AND USED IN THIS STUDY.

\begin{tabular}{|c|c|c|c|c|c|c|c|c|}
\hline \multirow[b]{2}{*}{ Reference } & \multicolumn{4}{|c|}{ MWCNTs properties } & \multicolumn{2}{|c|}{ Epoxy properties } & \multirow[b]{2}{*}{$\begin{array}{c}\text { Measurement } \\
\text { device for } K_{\mathrm{c}}\end{array}$} & \multirow[b]{2}{*}{$\underset{(\%)}{\text { Range of } V_{f}}$} \\
\hline & $\begin{array}{l}\text { Density } \\
\left(\mathrm{g} / \mathrm{cm}^{3}\right)\end{array}$ & $\begin{array}{c}K_{\mathrm{f}} \\
\left(\mathrm{W} \cdot \mathrm{m}^{-1} \mathrm{~K}^{-1}\right)\end{array}$ & $\begin{array}{c}\text { Length } \\
(\mu \mathrm{m})\end{array}$ & $\begin{array}{c}\text { Diameter } \\
(\mathrm{nm})\end{array}$ & $\begin{array}{c}K_{\mathrm{m}} \\
\left(\mathrm{W} \cdot \mathrm{m}^{-1} \mathrm{~K}^{-1}\right)\end{array}$ & $\begin{array}{l}\text { Density } \\
\left(\mathrm{g} / \mathrm{cm}^{3}\right)\end{array}$ & & \\
\hline$[11]$ & 2.09 & 3000 & $0.1-10$ & 10 & 0.22 & 1.24 & Hot Disk TPS 2500 & $0.01-0.59$ \\
\hline [12] & 2.09 & 3000 & 5 & $10-15$ & 0.29 & 1.15 & TCi Mathis Analyzer & $0.55-0.17$ \\
\hline [13] & - & - & $10-50$ & 20 & 0.12 & - & ASTM-E12 25-87 & $0.30-0.89$ \\
\hline [14] & - & - & $20-30$ & $10-20$ & 0.24 & - & Hot Disk TPS 2500 & $0.20-1.20$ \\
\hline [15] & - & - & - & - & 0.24 & - & Hot Disk AB & $0.06-0.17$ \\
\hline
\end{tabular}

\section{RESULTS AND DISCUSSION}

Figure 1 depicts the normalized experimental thermal conductivity data for epoxy/MWCNT nanocomposites that were taken from the technical literature and used in the present study. The data is shown as a function of filler volume fraction. Notably, data from [11], [13] and [15], and the initial data point from [14] appear to collapse approximately onto a single straight line.

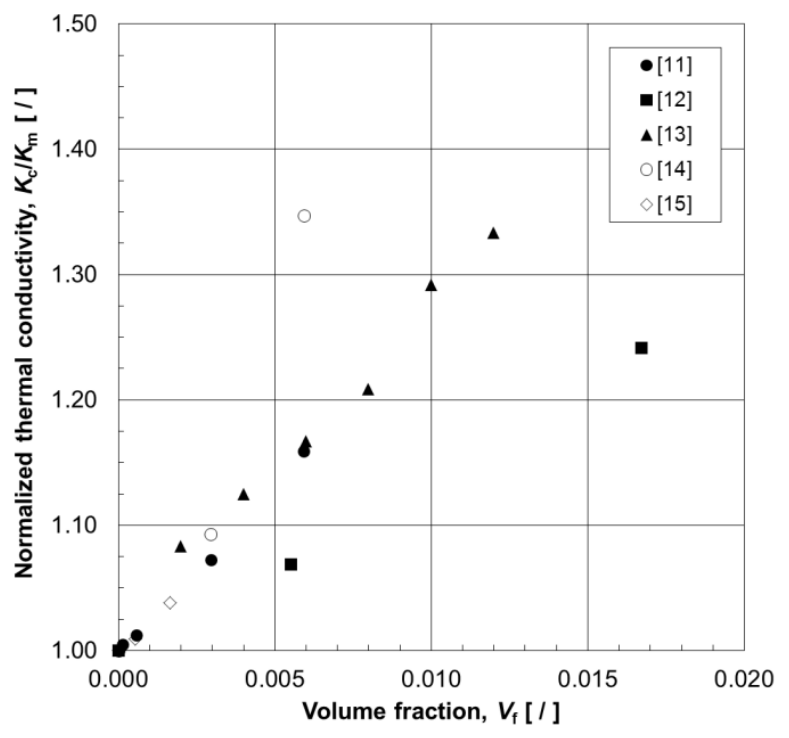

Figure 1. Normalized thermal conductivity of epoxy/MWCNT nanocomposites taken from technical literature.

An attempt was made to predict the experimental thermal conductivity data of nanocomposites shown in Fig.1. The models proposed by Maxwell [7], Hamilton and Crosser [8], Lewis-Nielsen [9] and Deng et al. [10] were employed for the predictions. Table 2 summarizes the parameter values that were used in this context. Figure 2 depicts the predicted values in terms of normalized thermal conductivity for the above models. Note that due to a lack of information on the filler morphology a filler straightness ratio $\theta$ of 0.7 was specified for the analysis, which assumes a MWCNT with moderate curvature.

Figure 2 indicates that the Hamilton and Crosser model overestimates the experimental data. This behavior can be explained by analyzing the empirical shape factor function, Eq.(3), which was derived for geometries that deviate from sphericity $(\gamma)$ by as low as 0.5 . In this study, the sphericity value was calculated to be 0.012 , which clearly indicates that
Eq. (3) was used outside of their study range. However, the Hamilton and Crosser model can be modified by determining an empirical shape factor expression that accounts for the high aspect ratio of CNT. The Deng et al. model predicts thermal conductivity values that are higher than the experimental data from literature. Nevertheless, the Deng et al. model yielded predictions that are closer to experimental data than the Hamilton and Crosser model. It should be noted that the model by Deng et al. is rather comprehensive in the sense that it accounts for various nanocomposite morphology factors, i.e., ITR, aspect ratio, size and geometry.

In contrast, the Maxwell and the Lewis-Nielsen models underestimate the literature data. The Maxwell model was developed for spherical fillers and ignored the effect of the ITR, the filler shape and aspect ratio. According to Nan et al. [18], the aspect ratio of CNT has a strong impact on the thermal conductivity of a composite. A large aspect ratio of CNT will minimize the amount of polymer matrix in between fillers, which results in an increased thermal conductivity by reducing the ITR as the probability of direct CNT contact increases [18]. Therefore, the Maxwell model cannot effectively predict fillers with high aspect ratios especially at higher volume fractions.

An attempt was made to adjust the predictive models for the two models closest to the experimental data, i.e. Deng et al. model and the Lewis-Nielsen model. The Deng et al. model prediction was modified by adjusting the filler straightness ratio $\theta$ while keeping other parameters constant. A straightness ratio of $\theta=0.4$ was implemented, which is lower than the initially assumed value of 0.7 . A lower $\theta$ signifies that the CNT dispersed in the polymer matrix are not straight but rather curved. In an experimental study, an effort could be made to quantify the CNT straightness in nanocomposite samples using e.g. transmission electron microscopy.

TABLE 2. PARAMETERS USED FOR THERMAL CONDUCTIVITY PREDICTIONS.

\begin{tabular}{llc}
\hline \multicolumn{1}{c}{ Parameter } & \multicolumn{1}{c}{ Value } & Reference \\
\hline Average $K_{\mathrm{m}}\left(\mathrm{W} \cdot \mathrm{m}^{-1} \cdot \mathrm{K}^{-1}\right)$ & 0.22 & \\
$K_{\mathrm{f}}\left(\mathrm{W} \cdot \mathrm{m}^{-1} \cdot \mathrm{K}^{-1}\right)$ & 3000 & Table 1 \\
Average length $(\mu \mathrm{m})$ & 17 & \\
Average diameter $(\mathrm{nm})$ & 16 & {$[9]$} \\
Shape coefficient $A$ & 8.38 & {$[9]$} \\
$V_{\mathrm{m}}$ & 0.52 & {$[16]$} \\
$K_{\mathrm{c}}^{33}$ & 3000 & {$[17]$} \\
$R_{\mathrm{k}}\left(\mathrm{m}^{2} \cdot \mathrm{K} \cdot \mathrm{W}^{-1}\right)$ & $8.30 \mathrm{E}-08$ & Assumption \\
$\theta$ & 0.7 & \\
\hline
\end{tabular}




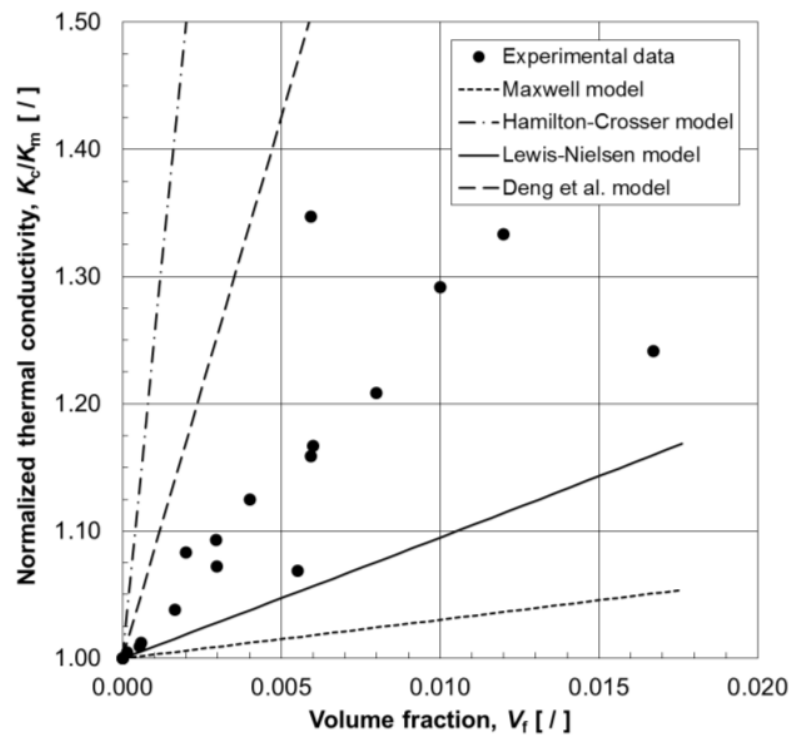

Figure 2. Normalized data for predicted thermal conductivity and experimental values as a function of filler volume fraction.

Similarly, the Lewis-Nielsen model prediction was adapted by changing the shape coefficient, $A$, as it is the most sensitive parameter in the model. Setting $A=26$ yielded values that matched the adjusted prediction based on the Deng et al. model. However, for the sake of clarity, $A$ was set to 24 so that the reader can distinguish between the two models shown in Fig.3. For the considered filler volume fraction, these findings indicate that the filler geometry and aspect ratio play a critical role for the accurate prediction of thermal conductivity of polymer nanocomposites as demonstrated by adjusting respective parameters for both the Deng et al. and LewisNielsen models.

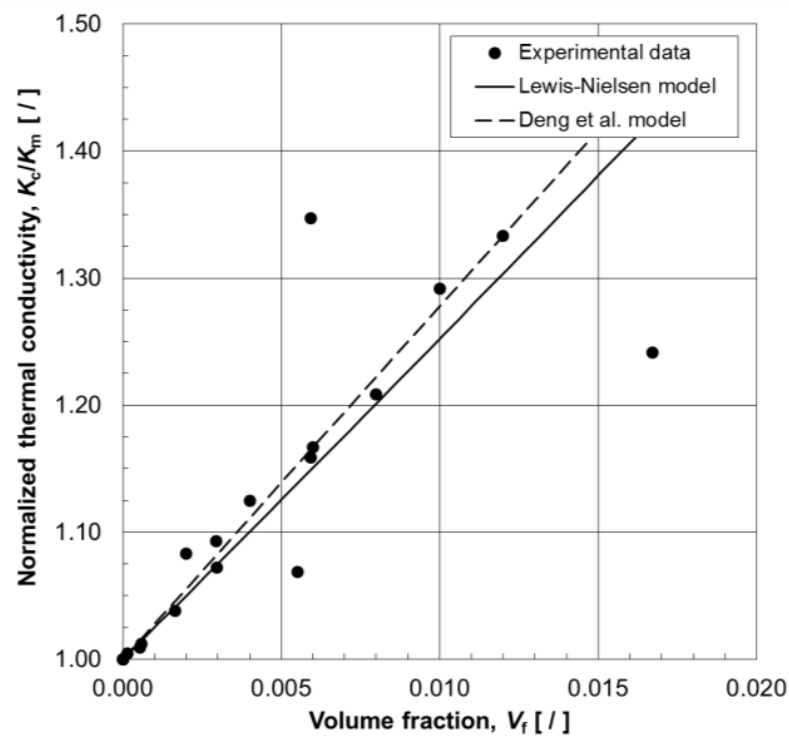

Figure 3. Normalized thermal conductivity predictions from the LewisNielsen and Deng et al. models with adjusted filler morphology parameters, compared to experimental data.

\section{CONCLUSIONS AND RECOMMENDATIONS}

In the present study, models for the prediction of the thermal conductivity of epoxy/MWCNT nanocomposites were examined. A set of prevalent analytical models and experimental data were selected from the technical literature. All models indicated an increase in thermal conductivity with increasing filler loading. For the range of filler volume fractions studied the following conclusions were derived.

- The Maxwell model strongly underestimated the thermal conductivity for epoxy/MWCNT nanocomposites. This model was developed for spherical fillers and is therefore limited in its ability to predict the thermal conductivity of high aspect ratio fillers.

- The Hamilton and Crosser model strongly overestimated the thermal conductivity of epoxy/MWCNT nanocomposites, which is due to a shape factor function that is incompatible with high aspect ratio CNT fillers.

- The Lewis-Nielsen model and the Deng et al. model yielded the most favorable predictions. Results from models could further be improved by adjusting the shape factor and filler straightness ratio, respectively.

- In general, it was observed that the parameters related to the filler morphology (e.g. aspect ratio, curvature) have a strong influence on thermal conductivity predictions. Predictions are less sensitive to the physical properties of the constituents such as their individual thermal conductivities.

\section{REFERENCES}

[1] F. Du, T. Kashiwagi, J.F. Douglas, R.H. Harris, J.R. Shields, and K.I. Winey, "Nanoparticle networks reduce the flammability of polymer nanocomposites," Nat. Mater., vol. 4, pp. 928-933, 2005.

[2] A.P. Mouritz, S. Feih, E. Kandare, Z. Mathys, A.G. Gibson, P.E. Des Jardin, S.W. Case, and B.Y. Lattimer, "Review of fire structural modelling of polymer composites," Compos. Part A-Appl. S., vol. 40, pp. 1800-1814, 2009.

[3] T. Kashiwagi, E. Grulke, J. Hilding, R. Harris, W. Awad, J. Douglas, "Thermal Degradation and Flammability Properties of Poly(propylene)/Carbon Nanotube Composites," Macromol. Rapid Comm., vol. 23, pp. 761-765, 2002.

[4] Q. Wu, W. Zhu, C. Zhang, Z. Liang and B. Wang, "Study of fire retardant behavior of carbon nanotube membranes and carbon nanofiber paper in carbon fiber reinforced epoxy composites," Carbon, vol. 48, pp. 1799-1806, 2010.

[5] Z. Han, A. Fina, "Thermal conductivity of carbon nanotubes and their polymer nanocomposites: A review," Prog. Polym. Sci., vol. 36, pp. 914-944, 2010.

[6] S.J.J. Nejad, "A review on modeling of the thermal conductivity of polymeric nanocomposites," e-Polymers, vol. 12, pp. 253c-288c, 2012.

[7] Y. Zhang, Y. Wang, C.M. Wang and Y. Gu, "Thermal conductivity of graphene and its polymer nanocomposites: A Review," Advanced Computational Nanomechanics, N. Silvestre, Eds. Chichester:Wiley, 2016, pp. 1-28.

[8] R.L. Hamilton and O.K. Crosser, "Thermal conductivity of heterogeneous two component systems," Ind. Eng. Chem. Fundamen., vol. 1, pp. 187-191, 1962.

[9] K. Pietrak and T.S. Wisniewski, "A review of models for effective thermal conductivity of composite materials. Journal of Power Technologies, vol. 95(1), pp. 14-24 14, 2015.

[10] L. Wang, C. Nan, F. Deng and Q. Zheng, "Effects of anisotropy, aspect ratio, and nonstraightness of carbon nanotubes on thermal conductivity of carbon nanotube composites," Appl. Phys. Lett., vol. 90, 021914, 2007. 
[11] V. Romano, C. Naddeo, L. Guadagno, and L. Vertuccio, "Thermal conductivity of epoxy resins filled with MWCNT and hydrotalcite clay: Experimental data and theoretical predictive modeling," Polym. Composite., vol. 36, pp. 1118-1123, 2015.

[12] C. Kostagiannakopoulou, E. Fiamegkou, G. Sotiriadis and V. Kostopoulos, "Thermal conductivity of carbon nanoreinforced epoxy composites," J. Nanomater., vol. 2016, 1847325, 2016.

[13] J. Jiao, Y. Cui and Y. Xia, "Improved thermal conductivity of epoxy composites prepared with a mixed filler of multiwalled carbon nanotubes and aluminum nitride particles," High. Perform. Polym., vol. 29, pp. 484-492, 2017.

[14] Y.S. Song and J.R. Youn, "Influence of dispersion states of carbon nanotubes on physical properties of epoxy nanocomposites," Carbon, vol. 43, pp. 1378-1385, 2005.

[15] F.H. Gojny, M.H.G. Wichmann, B. Fiedler, I.A. Kinloch, W. Bauhofer, A.H. Windle and K. Schulte, "Evaluation and identification of electrical and thermal conduction mechanisms in carbon nanotube/epoxy composites," Polymer, vol. 47, pp. 2036-2045, 2006.

[16] P. Kim, L. Shi, A. Majumdar and P.L. McEuen, "Thermal transport measurements of individual multiwalled nanotubes," Phys. Rev. Lett., vol. 87, 215502, 2001.

[17] S. Huxtable, D.G. Cahill, S. Shenogin, L. Xue, R. Ozisik, P. Barone, M. Usrey, M.S. Strano, G. Siddons, M. Shim, and P. Keblinski, "Interfacial heat flow in carbon nanotube suspensions, "Nat. Mater., vol. 2, pp. 731-734, 2003.

[18] C.W. Nan, G. Liu, Y. Lin, and M. Li, "Interface effect on thermal conductivity of carbon nanotube composites," Appl. Phys. Lett., vol. 85, pp. 3549-3551, 2004. 